\title{
Complex Regional Pain Syndrome
}

\author{
Gabor B. Racz ${ }^{1}$ and Carl E. Noe ${ }^{2}$ \\ ${ }^{1}$ Department of Anesthesiology, Pain Center, \\ Texas Tech University Health Sciences Center, \\ ${ }^{2}$ Department of Anesthesiology and Pain Management, \\ Eugene McDermott Center for Pain Mangement, \\ Univerisity of Texas Southwestern Medical Center, \\ USA
}

\section{Introduction}

Complex regional pain syndromes (CRPS) are pain syndromes characterized by pain out of proportion to an inciting injury, swelling, discoloration, stiffness, hyperhidrosis (sudomotor), temperature (vasomotor) and trophic changes. Also commonly seen are fine tremor and less often spasms involving upper and lower extremities. Dr. Silas Wier Mitchell described CRPS II, or causalgia, during the American Civil War. CRPS I was described about the end of the 19 ${ }^{\text {th }}$ century by Sudek (Sudek's atrophy). Evans described reflex sympathetic dystrophy (RSD). Numerous other terms used to describe similar syndromes include algodystrophy and shoulder- hand syndrome. Bonica described 3 stages of RSD. Roberts described sympathetically maintained pain.

\section{Diagnostic criteria}

Specific inclusion criteria are needed for research studies but from a clinical perspective, many patients seem to have a constellation of signs and symptoms of CRPS without meeting strict criteria. The diagnosis is made by the process of exclusion. While avoiding over diagnosing and over treatment, the patients need to be treated.

\section{Prognosis}

The prognosis for CRPS is highly variable and to a large extent is influenced by the treatment. Functional restoration and involving the patient in ongoing range of motion and resistive exercises is helpful. Timely pain relief and interventional pain procedures, as well as psychological support, are important. Patients need to be followed closely and treatments adjusted accordingly. Timely and appropriate referral to experienced pain physicians that are able to offer multimodal therapies may prevent costly delays and complications. 


\section{Theories of mechanisms}

Multiple possible mechanisms exist for CRPS including psychological, inflammatory, vascular, neurogenic and combinations of several mechanisms. Debate regarding definitions of neuropathic pain has led to the notion that CRPS may not be neuropathic pain. Psychogenic pain could be construed as being "pain arising as a direct consequence of a lesion or disease affecting the somatosensory system" but few would think of it as neuropathic pain which should be treated with anticonvulsants.

CRPS II is generally agreed to be caused by an injury to a peripheral nerve. CRPS 1 is caused by a lesion in or injury to a small nerve or multiple small nerves. It is difficult to accept that it is not neuropathic pain since it resembles CRPS II so closely. Denial of care based on psychological explanations is neither reasonable nor justifiable yet in rare instances pain can be of psychological origin. Commonly the onset of CRPS is 1- 3 months after the injury.

\section{History}

The diagnosis is made by process of exclusion following history of pain that is out of proportion to an injury or period of immobilization. Swelling, temperature asymmetry, stiffness, atrophy, hair, skin nail, bone changes. Tremor or spasms and asymmetry in sweat function are all potential signs. It is important to remember that many injuries are associated with pain, discoloration and swelling without being CRPS. Infection and other causes of inflammation are sometimes mistakenly thought to be CRPS. A number of patients have CRPS symptoms following stroke and classifying this as central pain or CRPS is problematic.

\section{Physical exam}

Observation of upper extremity guarding or antalgic gait for lower extremity is important. Range of motion of affected joints is particularly important as many patients develop permanent stiffness without analgesia for specific range of motion therapy. Discoloration or asymmetrical coloration, swelling, atrophy and allodynia are other physical findings. The allodynia may be tactile or cold induced.

\section{Diagnostic tests}

Bone scans, sweat tests and sympathetic blocks have been used but the diagnosis is a clinical one and can be made without confirmatory tests. Thermography has been used, but more commonly, the documentation of temperature differences is adequate. Early on in the evolution of the condition there may increased temperature and later reduction with the increased sympathetic activity. Three phase bone scan often show corresponding changes. Comparing contra lateral x-ray images can show osteopenia in the involved area. EMG usually does not change from the CRPS but may show nerve injury.

\section{Differential diagnosis}

While it important to be vigilant in diagnosing CRPS, as is important to avoid misdiagnosis and over- diagnosis. Many patients have "pain out of proportion", swelling and 
discoloration after injuries and will improve within a month with usual therapeutic interventions.

Infection is always a concern after surgery or other penetrating trauma. Other causes of acute inflammation, swelling and discoloration need to be considered such as malignancy, deep venous thrombosis as well as peripheral nerve entrapment, peripheral neuropathy and other neuropathic pains.

\section{Stages}

3 stages of RSD have been described however it is unclear that staging has much value regarding decision making.

\section{Timing}

Much has been made about early sympathetic blocks and failure to diagnose early. There is no data to support "emergent" sympathetic blocks and some patients have a favorable natural history.

\section{Spreading}

Pain from CRPS can spread, in rare instances, proximally and contra- laterally. (Shah, Racz) Lower extremity pain can spread to upper extremities and vice versa.

\section{Bone loss}

Osteopenia and fractures can occur in severe cases and aquatic therapy is useful to rehabilitate these patients.

\section{Natural history}

The natural history of CRPS 1 is variable but in an interesting report, approximately $25 \%$ of patients that had Colles' fractures developed signs of CRPS. (Atkins) Approximately $40 \%$ of these patients improved in 6 months. This suggests that mild cases may not require extensive treatment. Not treating the patients early can be problematic if the condition worsens. Appropriate examination and follow up is important where the disease can take a benign course. Patients obtain information on the Internet that is usually about catastrophic cases that needs to be dealt with by educating patients in an appropriate and caring manner where therapy is timely yet one can avoid catastrophizing based on inaccurate information.

\section{Dogma}

Much of "standard care" is not evidence based, but good outcome based. Additionally, it is based on physician experience and the outcome is superior in the hands of better-trained physicians. As new information becomes available, dogma can be weeded out and treatments based on randomized controlled trails can be incorporated into treatment guidelines. 


\section{Cases}

One lady had not worn high-heeled shoes for a long time and then wore a pair for several hours at an event. She developed classic signs and symptoms of RSD. She experienced profound analgesia with sympathetic blockade and the condition resolved completely.

Another case was a woman who had a paper cut on her distal index finger on the job. She had classic signs and symptoms of CRPS, which resolved with a series of blocks. Both of these cases were challenged by insurance companies since the inciting injury was so minor but both patients were legitimate. The point is that physicians caring for these patients must be willing to serve as advocates for the patient even in an environment of cost containment. We have to be mindful of our "report cards" but not at the expense of a patient's outcome.

\section{Overmedication pain syndrome (OPS)}

Approximately 20 years ago, a movement began to improve the quality of pain care for cancer patients worldwide. The WHO analgesic ladder was promoted for cancer pain and then it was applied for other types of pain. Many patients are now taking large doses of opioid for chronic pain.

Overmedication pain syndrome is characterized by a chronic treatment program consisting of high doses of multiple analgesic medications without associated functional productivity and psychological coping ability.

Opioids are the most important class of drugs in pain management; however, it is clear that they are two edged swords and overmedication with opioids and other drugs classes have become a problem. Abuse may not be the largest problem. Lack of efficacy, unintended overdose, diversion, development of drug dependence, habituation and resistance to recovery and other unintended consequences may be more common.

Opioid induced hyperalgesia is a real clinical phenomenon and may be a subtle barrier to analgesia in many patients. Pain that is only incrementally responsive to opioid is also common.

Pseudo-addiction is defined in behavioral terms which are similar to addiction but related to pain and not addiction. The problem is that there are not good means to differentiate behaviors between the setting of pain and the setting of addiction.

Some have reported a lack of data to support doses of opioid over $200 \mathrm{mg}$ / day of morphine equivalents. Also, there are no long term randomized controlled trials of opioid versus placebo. Additionally, fracture rates have been reported to be increased in patients on doses above $100 \mathrm{mg} /$ day. (Sullivan) Overdose rates have been reported to increase above 50 $\mathrm{mg} /$ day. (Dunn) Drug interactions with other medications, reported and unreported to the treating physician, have been causes of fatalities.

Urine drug testing, opioid contracts and extensive documentation guidelines fail to help answer the clinical question: is the dose just too high?

Patients who are taking opioids chronically should be considered for an evaluation for a lack of meaningful efficacy, fall and fracture risk and overdose risk. An interdisciplinary evaluation may be a way to accomplish these objectives. Patients who are clearly doing well 
may be less likely to accept dose reductions. Patients who are working or similarly productive and are without signs of poor coping and physical disability may need to continue taking the effective dosages. On the other hand, patients, who have been on stable doses for a long time may need age related dose reductions.

Washington State has new guidelines limiting the dose of opioid to $120 \mathrm{mg} /$ day of morphine equivalents. Patients, who require doses above this level, are guided to seek a pain management consultation. The purpose and intervention of a medical pain management consultation is unclear. The practitioner doing the evaluation needs to have additional training and qualification as well as be informed and knowledgeable in treatment options in addition to opioid management.

JCAHO, Press Gainey and other organizations have changed the environment with respect to patient rights regarding pain. In the past, if a patient wasn't happy with their opioid dose, their recourse was limited. Now, patient satisfaction is used as a factor to determine healthcare provider's compensation. The implication is that patients can pressure providers to prescribe more opioid, which is dangerous for patients and providers.

Regulators have become more active due to the increased rate of diversion and its consequences. However, the accidental overdose rate increase is even more concerning.

Most drugs have dose limits. For example, antibiotics and drugs for hypertension are increased to upper limits but there are limits. Perhaps it is time to limit doses of opioids regardless of pain severity for patients with non palliative care pain syndromes and find another way to treat the patient.

Other drugs classes that are problematic include benzodiazepines, muscle relaxers, sleeping pills and even anticonvulsants and antidepressants. Benzodiazepines are not prominent in the pain literature as analgesics. Baclofen and tizanidine are probably the first line muscle relaxers of choice. Hypnotic drugs are used too often for chronic sleep disturbances without sleep hygiene treatment or other medications which are better for long term use. Anticonvulsant use for chronic pain has exploded as opioids have. Antidepressants, even those not associated with analgesia, are prescribed for pain.

The costs of these drugs are significant and usually of incremental benefit. Most patients with chronic pain go without an interdisciplinary evaluation and many who receive an evaluation do not complete treatment with cognitive behavioral therapy, education and conditioning physical therapy. Treatment goals are frequently not established and some patients just go through the motions and are considered as a treatment failure. There is very little evidence for the multidisciplinary and physical therapy based treatments specifically for CRPS. Reimbursement has suffered for these kinds of therapies.

The cognitive effects and psychological effects of chronic opioid treatment are not well known.

Testosterone levels in males are known to decrease with chronic opioid administration.

It is proposed that patients with chronic pain have a short term trial of low dose opioid to access functional improvement before a treatment plan is finalized. Blinding patients to their drug and dose may be very helpful but has its critics on ethical and regulatory grounds.

Patients who are on doses above $50 \mathrm{mg} /$ day of morphine equivalents need to have access to interdisciplinary pain and addictionology evaluations and treatment if needed. Treatment goals should include dose reduction to below $200 \mathrm{mg} /$ day of morphine equivalents for those 
taking more than that. Intermediate term treatment goals for patients taking less than $200 \mathrm{mg} /$ day should strive for less than $100 \mathrm{mg} /$ day and patients taking less than $100 \mathrm{mg} /$ day, $50 \mathrm{mg} /$ day.

There is no data to support this approach but there was no data 20 years ago to support using the WHO analgesic ladder for headaches, fibromyalgia, back pain or any other condition. Data for limited doses of opioid for arthritis and neuropathic pain exists and prescribing for opioid responsive pain should not be overly scrutinized by regulators. Never the less, diversion, addiction, opioid induced hyperalgesia and other adverse events associated with opioids need to be avoided more effectively before the first prescription is written.

Many patients in drug treatment programs were initially treated with opioid for perfectly legitimate pain. The patient and the doctor may not be the biggest problems. The biggest problem may be the drug and the dosage.

\section{Treatment guideline history}

In 1994, the International Association for the Study of Pain (IASP) revised the terminology from RSD and causalgia to CRPS type I and II. 15 years ago we proposed an analgesic ladder for CRPS /RSD which included 3 steps. (Racz) Since then, well-respected groups have advanced other guidelines. (Van Eijs) (Stanton-Hicks)

Our initial proposal was:

Step 1. TENS, opioids, topicals, Tricyclic antidepressants, supportive psychotherapy, vocational rehabilitation, patient education, physical therapy and occupational therapy

Step 2. Regional or sympathetic block, evaluation and treatment of the emotional component of pain, IV regional block, peripheral block-infusion, carbamazepine, baclofen, clonidine, corticosteroid, NSAID, mexiletine, other drug trials

Step 3. Sympathectomy/sympatholysis, peripheral nerve decompression, lysis, continuous local anesthetic infusion epidural and or regional for five to seven days, Spinal Cord Stimulation (SCS), Peripheral Nerve Stimulation (PNS), intrathecal/epidural analgesia.

At that time, little data existed to guide treatment and the initial analgesic ladder was based on opinion. Since that time, additional data has been produced leading to modifications to the analgesic ladder. This is categorically not intended to estabish a standard of care since data to do such is inadequate. Rather, our intention is to share our beliefs in hopes of helping patients with this disorder.

\section{New principles and information}

Our current analgesic ladder promotes several concepts:

1. Interdisciplinary pain treatment is recommended rather than multidisciplinary care which tends to be fragmented. Interdisciplinary treatment specifically provides coordinated medical care, education, cognitive behavioral therapy for pain, physical therapy and outcome documentation by the interdisciplinary team. Patients who receive care at different clinics for each component of care by a group of providers who do not meet on a weekly basis nor document comprehensive outcomes are not receiving interdisciplinary pain management. 
2. Interdisciplinary care is not isolated from medical pain management. Analgesic treatments are necessary to provide pain relief and allow functional restoration.

3. The course of an individual patient is highly variable and adjustments to the treatment plan should be made in a highly flexible manner.

4. Limiting opioid doses to below $200 \mathrm{mg}$ / day morphine equivalents

5. Numerous randomized controlled trials have been performed since our initial analgesic ladder was proposed and these findings are incorporated.

6. However if there is treatment failure and functional restoration failure the patient needs to be referred to centers or individuals with recognized experience to be specialists in the field.

Sympathetic blocks have been recommended early on in the management of the disorder but little data exists to support this practice. Only recently has any data from a randomized controlled trial been published to demonstrate efficacy of sympathetic blockade. (Meier) Spinal cord stimulation has been shown to produce significant analgesia even after 5 years of treatment. (Klemer) Cortical stimulation has been shown to have some benefit. (Velasco) Deep brain stimulation has been shown to be ineffective. Vitamin $C$ has been studied by multiple investigators for the prevention of CRPS and has some effect. (Besse) Intravenous magnesium has been reported to be effective in an initial study. (Collins) Clodronate has been shown to be partially effective. (Varenna) Mirror therapy has been reported to have benefit in stroke patients with CRPS. (Cacchio) Multicenter comparison of spinal cord stimualtion and peripheral nerve stimulation showed that PNS is more effective than SCS but the best outcome was where both modalities were utilized. (Calvillo)

Intravenous regional anesthesia with the addtion of vasodilators such as phentolamine, reserpine and bretylium allow manipulation of hands without post procedure edema and speed up functional restoration without the pain associated with physical therapy. (Heavner, Calvillo, Racz)

An evidenced based review endorses bisphosphonates (alendronate, pamidronate, clodronate), corticosteroid, gabapentin, physiotherapy and psychotherapy/relaxation techniques as treatments. (Baron) Additionally intrathecal baclofen for associated dystonia and spinal cord stimulation for refractory caes are recommended. Topical DMSO and sympathetic blocks are not strongly recommended. Intravenous regional blocks with guanethidine are not recommended as specific treatment (Van Eijs)

\section{Treatments to avoid}

Amputation is less common nowadays because it was rarely effective and usually resulted in a phantom pain plus different pain of greater severity

IV regional with guanethidine has been shown to be ineffective in several studies as sole agent.

Deep brain stimulation has been shown to be ineffective.

High dose opioid should be avoided if possible due to possible opioid induced hyperalgesia, addiction, diversion risk and over-dosage.

\section{Proposed treatment}

\section{Step 1.}

Screening for substance abuse, affective disorders and disability Education 
Physical therapy

Occupational therapy

Vocational rehabilitation

Topical lidocaine for allodynia

Tricyclic antidepressants

Gabapentin

Tramadol

Opioid doses limited to less than 200mg morphine equivalents per day and below $50 \mathrm{mg} /$ day if possible

Corticosteroid

\section{Step 2.}

Interdisciplinary pain evaluation including psychological testing (MMPI-RF) and treatment (cognitive behavioral therapy, group psycho educational therapy and psychotropic medication management, addictionology, physical and occupational therapy, in a coordinated goal directed, outcome documenting rehabilitation program)

Sympathetic block

IV Regional block

Peripheral block

Other drug trials

Step 3.

Spinal cord stimulation

Sympathectomy/sympatholysis

Peripheral nerve stimulation

Peripheral nerve decompression/lysis

Intrathecal/epidural analgesia

\section{Interdisciplinary care}

Interdisciplinary pain management is a term that is poorly understood. It is best reserved to describe a team of healthcare professionals led by a physician and including a psychologist and physical therapist at a minimum. A care team of multiple physicians from different specialties is not an interdisciplinary team for pain management nor is a psychologically based treatment program in isolation from medical pain management. Cognitive behavioral therapy, education and functional rehabilitation must be provided in an interdisciplinary pain care model in addition to medical pain management therapies. Case management, psychiatry, outcome database management, nursing, vocational rehabilitation, occupational therapy, medical direction and program direction and administrative support are key disciplines to include in a mature pain program. Nutrition, chaplaincy and other medical specialties are needed for tertiary programs.

\section{Conclusion}

Complex regional pain syndrome is a challenging pain problem that frequently requires a comprehensive interdisciplinary assessment and treatment plan. Until a mechanism is discovered and a specific treatment for the syndrome is developed, an interdisciplinary approach, including pharmacologic and interventional pain management in a step wise fashion, will likely remain as the best route to follow. 


\section{References}

Atkins, R.M., Duckworth, T., Kanis, J.A.: Algodystrophy following Colles' Fracture.Journal of Hand Surgery (British Volume, 1989) 14B: 161-164

Baron, R., Naleschinski, D., Hullemann, P., Mahn, F.: Complex Regional Pain Syndrome: A neuropathic disorder? Pain 2010- An updated Review: Refresher Course Syllabus. IASP Press p. 109-117. 2010

Besse, J., Gadeyene, S., Galand-Desme, S., et.al: Effect of vitamin C on prevention of complex regional pain syndrome in foot and ankle surgery. Foot and Ankle Surgery 15:179182, 2009

Cacchio, A., De Blasis, E., De Blasis, V., at.al.:Mirror Therapy in Complex Regional Pain Syndrome Type 1 of the Upper Limb in Stroke Patients. Neurorehabilitation and Neural Repair 23: 792-799, 2009.

Calvillo O, Racz GBN, Diede J, Smith K: Neuroaugmentation in the treatment of complex regional pain syndrome of the upper extremity. Acta Orthopaedica Belgica, 64-1, 57-63, 1998.

Collins, S., Zuurmond, W.W.A., de Lange, J.J., et.al.: Intravenous Magnesium for Complex Regional Pain Syndrome Type 1 (CRPS 1) Patients: A Pilot Study. Pain Medicine 10:930-940, 2009.

Dunn KM, Saunders KW, Rutter CM, et al.: Opioid prescriptions for chronic pain and overdose: a cohort study. Annals of Internal Medicine 2010; 152:85-92

Heavner JE, Calvillo O, Racz GB: Thermal grill illusion and complex regional pain syndrome Type I Reflex Sympathetic Dystrophy. Regional Anesthesia 22(3): 257259, 1997.

Klemer M.A., de Vet, H.C., Barendse, G.A.M., et.al.: Effect of spinal cord stimulation for chronic complex regional pain syndrome Type I: five-year follow-up of patients in a randomized controlled trial. Jounal of Neurosurgery 108:292-298, 2008.

Meier, P.M., Zurakowski, D., Berde, C. B., Sethna, M.B.: Lumbar sympathetic blockade in Children with Complex Regional Pain Syndromes. Anesthesiology 2009, 111: 37280.

Racz, Gabor B., Heavner, James E., Noe, Carl E.: Definitions, classification and Taxonomy: an overview Sympathetic pain syndromes: reflex sympathetic dystrophy and causalgia. Physical Medicine and Rehabilitation: State of the Art Reviews Vol 10 No 2 June 1996 Hanley and Belfus, Philadelphia

Shah RV, Racz GB. Recurrence and spread of complex regional pain syndrome due to distant site surgery: a case report. Am J Orthop (Belle Mead NJ). 2006 Nov; 35(11): 523-6.

Stanton-Hicks, M., Baron, R., Boas, R., et.al: Complex regional pain syndromes: guidelines for therapy. Clinical Journal of Pain 14:155-166, 1998.

Sullivan, M.D.: Who gets high dose opioid therapy for chronic non-cancer pain? Pain 151:567-568, 2010.

Van Eijs, F., Stanton -Hicks, M., Van Zundert, J., et. al.: Complex Regional Pain Syndrome. Pain practice 11:70-87, 2010. 
Varenna, M., Zucchi, F., Ghiringhelli, D., et.al.: Intravenous clodronate in the treatment of reflex symathetic dystrophy syndrome. Journal of Rheumatology 27:1477-83, 2000

Velasco, F., Carrillo-Ruiz, J.D., Castro, G., et.al.: Motor cortex stimulation applied to patients with complex regional pain syndrome. Pain 147:91-98, 2009. 


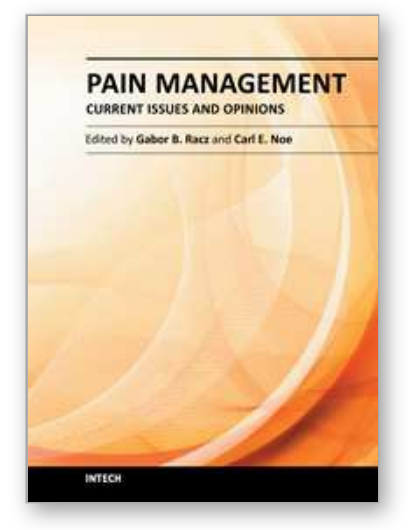

\author{
Pain Management - Current Issues and Opinions \\ Edited by Dr. Gabor Racz
}

ISBN 978-953-307-813-7

Hard cover, 554 pages

Publisher InTech

Published online 18, January, 2012

Published in print edition January, 2012

Pain Management - Current Issues and Opinions is written by international experts who cover a number of topics about current pain management problems, and gives the reader a glimpse into the future of pain treatment. Several chapters report original research, while others summarize clinical information with specific treatment options. The international mix of authors reflects the "casting of a broad net" to recruit authors on the cutting edge of their area of interest. Pain Management - Current Issues and Opinions is a must read for the up-to-date pain clinician.

\title{
How to reference
}

In order to correctly reference this scholarly work, feel free to copy and paste the following:

Gabor B. Racz and Carl E. Noe (2012). Complex Regional Pain Syndrome, Pain Management - Current Issues and Opinions, Dr. Gabor Racz (Ed.), ISBN: 978-953-307-813-7, InTech, Available from:

http://www.intechopen.com/books/pain-management-current-issues-and-opinions/complex-regional-painsyndrome

\section{INTECH}

open science | open minds

\section{InTech Europe}

University Campus STeP Ri

Slavka Krautzeka 83/A

51000 Rijeka, Croatia

Phone: +385 (51) 770447

Fax: +385 (51) 686166

www.intechopen.com

\section{InTech China}

Unit 405, Office Block, Hotel Equatorial Shanghai

No.65, Yan An Road (West), Shanghai, 200040, China

中国上海市延安西路65号上海国际贵都大饭店办公楼 405 单元

Phone: +86-21-62489820

Fax: +86-21-62489821 
(C) 2012 The Author(s). Licensee IntechOpen. This is an open access article distributed under the terms of the Creative Commons Attribution 3.0 License, which permits unrestricted use, distribution, and reproduction in any medium, provided the original work is properly cited. 